\title{
GLOBAL SOLUTIONS WITH A SINGLE TRANSONIC SHOCK WAVE FOR QUASILINEAR HYPERBOLIC SYSTEMS
}

\author{
Fumioki Asakura \\ Dedicated to Professor Kôji Kubota on his sixtieth birthday
}

\begin{abstract}
We shall study global solutions containing a single transonic shock wave for the general quasilinear hyperbolic system $U_{t}+F_{x}=G$. The presence of $G$ brings about secondary waves. We shall single out the amount of such waves along each characteristic field and show that global-in-time solutions exist provided the total variation of $U_{0},\|G\|_{1}$, and the total amount of secondary waves along the transonic characteristic are sufficiently small.
\end{abstract}

\section{Introduction}

We study the Cauchy problem for the general quasilinear hyperbolic system of the form:

$$
\begin{aligned}
\frac{\partial}{\partial t} U+\frac{\partial}{\partial x} F(U) & =G(x, U), & & (x, t) \in R \times R_{+}, \\
U(x, 0) & =U_{0}(x), & & x \in R .
\end{aligned}
$$

Here $U={ }^{t}\left(u_{1}, u_{2}, \ldots, u_{n}\right)$ is a vector function which takes on values in an open set $\Omega \subset R^{n} ; F(U)$ and $G(x, U)$ are smooth maps from $\Omega$ to $R^{n}$ and from $R \times \Omega$ to $R^{n}$, respectively. We assume that the system (1) is strictly hyperbolic, which means that the Jacobian matrix $F^{\prime}(U)$ has the $n$ real distinct eigenvalues

$$
\lambda_{1}(U)<\lambda_{2}(U)<\cdots<\lambda_{n}(U), \quad U \in \Omega .
$$

Let $R_{j}(U)$ denote the right eigenvector of $F^{\prime}(U)$ corresponding to $\lambda_{j}(U)$. We also assume that each characteristic field is genuinely nonlinear in the sense of Lax [7]:

$$
R_{j} \cdot \operatorname{grad} \lambda_{j} \neq 0 \text { for } U \in \Omega, \quad 1 \leq j \leq n .
$$

Since solutions to a quasi-linear hyperbolic system become singular in general after a finite time (see John [6] and Lax [8]), we study the solution which satisfies the equations in a weak sense; we say that a locally integrable vector function $U(x, t)$ is a weak solution if the correspondence $t \mapsto U(*, t) \in L_{\text {loc }}^{1}(R)$ is weakly continuous and the following integral identity holds

$$
\iint_{R \times R_{+}}\left\{U \cdot \phi_{t}+F(U) \cdot \phi_{x}+G(x, U) \cdot \phi\right\} d x d t+\int_{R} U_{0}(x) \cdot \phi(x, 0) d x=0
$$

for every $\phi \in \operatorname{Lip}_{\mathrm{c}}\left(R \times R_{+}\right)$.

If $G(x, U) \equiv 0$, the equations constitute a system of conservation laws, and the existence of weak global solutions to these systems was first established by Glimm

Received March 15, 1996, revised September 5, 1996. 
[3]. He constructed difference approximations in an ingenious way and showed that a subsequence converges to a weak solution provided the total variation $\operatorname{TV}\left(U_{0}\right)$ is sufficiently small. The crucial estimate for the proof is to get a uniform bound of $\operatorname{TV}\left(\left(U_{h}(*, t)\right)\right.$, the total variation in $x$ of approximate solutions. He showed that as time increases the total variation increases and decreases by the interaction of simple waves which constitute the approximate solutions and proved that the total amount of such interaction is $O(1)\left(\mathrm{TV}\left(U_{0}\right)\right)^{2}$.

For general quasilinear systems (1), the term $G(x, U)$ acts as a source term whose effects propagate at zero speed. Studies based on the Glimm method were carried out by Liu [12] who constructed difference approximations combining solutions to the classical Riemann problem and steady state solutions. In this case, besides the interaction of simple waves constituting approximate solutions, the secondary interaction, i.e., the interaction of simple waves and steady state solutions occurs and produces a certain amount of the secondary wave (Glimm et al. [5]). Let us define the quantity

$$
G(x)=\max \left\{|G(x, U)|+\left|G_{U}^{\prime}(x, U)\right|: U \text { in a small neighborhood of } U_{0}(x)\right\} .
$$

When the characteristic speeds are away from zero and the $L^{1}$-norm of $G(x)$ is finite, the interaction of a single wave and the source term is finite. Liu proved that the total amount of secondary wave produced is $O(1) \mathrm{TV}\left(U_{0}\right)$ times $\|G\|_{1}$. Hence, if $\operatorname{TV}\left(U_{0}\right)$ and $\|G\|_{1}$ are sufficiently small, global solutions exist and these solutions are stable in the sense that they converge to non-interacting wave patterns. If one of the characteristic speeds can be zero (the flow is transonic), new phenomena occur: we have a shock wave whose propagation speed is zero (a standing shock wave); the interaction of a standing shock wave and the source term may produce an infinite amount of secondary waves, because the standing shock wave and the effects of the source term propagate at the same speed and may interact for infinite time. Actually, in the transonic 1-D flow along the contracting duct, a standing shock wave is dynamically unstable and stable along the expanding duct (Liu [13]). When (1) is a single equation, Liu discussed an intrinsic approach to these phenomena [15].

We shall concentrate on solutions to (1) containing a single transonic shock wave and study in particular the secondary interactions. We single out the total amount of secondary interactions involving the single transonic shock wave and study conditions under which such an amount is uniformly bounded, which implies the global existence and stability of solutions.

We assume that the $p$-th characteristic speed alone can be zero. There exists $\delta>0$ such that

$$
\left|\lambda_{j}(U)\right| \geq \delta, \quad j \neq p, U \in \Omega
$$

and

$$
\mathcal{N}_{p}=\left\{U \in \Omega: \lambda_{p}(U)=0\right\} \neq \phi .
$$

We shall construct a solution in which the strength of the single transonic shock wave is $O(1) \alpha_{*}$ and the strengths of the other waves (weak waves) is $\ll \alpha_{*}$. In this case, the secondary interactions involving the $j$-th waves $(j \neq p)$ and weak $p$-waves are estimated in the same way as Liu [12] and only the secondary waves produced by the transonic waves remain. We first review, in Section 2, the definition and basic properties of steady state solutions. In Section 3, we construct approximate solutions 
using the scheme introduced in Liu $[12,13]$. In Section 4 we study the local interaction between weak waves and the single transonic shock wave. Let $\Delta$ denote an interaction diamond where the single strong shock wave enters. Let $h$ denote the mesh length, $G$ the maximum of $G(x)$ in $\Delta$, and $\sigma$ the speed of the transonic shock wave which enters $\Delta$. We shall show that the amount of waves produced by the local interaction is estimated by Glimm's quadratic term, $G h / \alpha_{*}^{2}$ times the amount of approaching waves and $\sigma G h / \alpha_{*}$ where the last term is the amount of secondary waves involving the transonic shock wave. In Section 5, we introduce a new Glimm potential and estimate the total amount of interaction in the presence of the transonic shock wave. The local interaction estimates indicate that the total amount of the secondary wave produced by the transonic wave is

$$
\sum_{n \geq 0} \frac{\left|\sigma_{n}\right| G_{m(n)} h}{\alpha_{*}}
$$

where the single strong shock wave enters $\Delta_{m(n), n}$ at $t=n k$ with speed $\sigma_{n}$. Since this is merely a time-dependent estimate, the above quantity (6) can become infinite, even if $\operatorname{TV}\left(U_{0}\right)$ and $\|G\|_{1} / \alpha_{*}^{2}$ are small. However, we shall show in Section 6 certain conditions which guarantee a uniform bound of (6) and hence the existence of global solutions.

\section{Steady state solutions}

Solutions to (1) are called steady state solutions if they do not depend on $t$. They satisfy the system of differential equations

$$
\frac{d}{d x} F(U)=G(x, U), \quad x \in R .
$$

Let $L_{j}(U)$ be a left eigenvector corresponding to $\lambda_{j}(U)$, chosen so that

$$
L_{j}(U) R_{j}(U)=1, \quad L_{j}(U) R_{k}(U)=0(j \neq k) .
$$

When $U \notin \mathcal{N}_{p}$, equation (7) is expressed as

$$
\frac{d U}{d x}=\sum_{j=1}^{n} \frac{g_{j}(x, U)}{\lambda_{j}(U)} R_{j}(U), \quad x \in R,
$$

where $g_{j}(x, U)=L^{j}(U) G(x, U)$. If the $L^{1}$-norm of the quantity $G(x)$ is sufficiently small compared with $\left|\lambda_{p}(U)\right|$, there exists a unique steady state solution for any given $U(0) \in \Omega$. Let $C: x=x(t)$ be a $C^{1}$-curve. We consider a weak solution of the form

$$
U(x, t)= \begin{cases}U_{L}(x), & x<x(t), \\ U_{R}(x), & x>x(t) .\end{cases}
$$

Proposition 2.1. Let $U_{L}(x)$ and $U_{R}(x)$ be steady state solutions, and $U(x, t)$ be defined as above. Then $U(x, t)$ is a weak solution in the sense (3) if and only if it satisfies the Rankine-Hugoniot condition

$$
\dot{x}(t)\left\{U_{R}(x(t))-U_{L}(x(t))\right\}=F\left(U_{R}(x(t))\right)-F\left(U_{L}(x(t))\right) .
$$


The proof is obvious.

We say that $\bar{U}(x)$ defined by

$$
\bar{U}(x)= \begin{cases}\bar{U}_{1}(x), & x<0 \\ \bar{U}_{2}(x), & x>0\end{cases}
$$

is said to be a p-standing shock wave if

1. $\bar{U}_{1}(x)$ and $\bar{U}_{2}(x)$ are steady state solutions,

2. $F\left(\bar{U}_{1}(0)\right)-F\left(\bar{U}_{2}(0)\right)=0$ (the Rankine-Hugoniot condition), and

3. $\lambda_{p}\left(\bar{U}_{2}(0)\right)<0<\lambda_{p}\left(\bar{U}_{1}(0)\right)$ (the Lax entropy condition).

The above proposition says that $\bar{U}(x)$ is a weak solution. Standing shock waves are easily constructed: first we choose a constant vector $U_{1}$ close to $\mathcal{N}_{p}$ satisfying $\lambda_{p}\left(U_{1}\right)>0$. Then we can find a unique constant vector $U_{2}$ on $\mathcal{S}_{p}\left(U_{1}\right)$, namely the shock curve issuing from $U_{1}$ satisfying $\sigma\left(U_{1}, U_{2}\right)=0$ and $\lambda_{p}\left(U_{2}\right)<0$. Thus, solving the equations (7) with initial data $U_{1}, U_{2}$, respectively, we have the steady state solutions $\bar{U}_{1}(x), \bar{U}_{2}(x)$, respectively, and obtain a standing $p$-shock wave by (11).

Let $\bar{U}(x)$ be a standing shock wave, and let $\alpha_{*}$ denote the strength of $\left\langle U_{1}(0), U_{2}(0)\right\rangle$. In this paper, we confine ourselves to the initial data which are the perturbation of $\bar{U}(x)$ in the total variation norm

$$
\begin{gathered}
U_{0}(x)= \begin{cases}\widetilde{U}_{1}(x), & x<0, \\
\widetilde{U}_{2}(x), & x>0,\end{cases} \\
\sum_{j=1,2} \operatorname{TV}\left(\widetilde{U}_{j}(x)-\bar{U}_{j}(x)\right) \ll \alpha_{*} .
\end{gathered}
$$

\section{Construction of approximate solutions}

Now we construct approximate solutions containing a relatively strong $p$-shock wave. We distinguish this single $p$-shock wave emerging at $x=0$ from other waves and call it the single strong shock wave; other waves are called weak waves.

Approximate solutions are constructed by the random choice scheme. Here we adopt a generalization of the Glimm scheme introduced in Liu [13].

Now we describe the difference scheme. Let $h, k$ be mesh lengths satisfying the stability condition

$$
\frac{h}{k}=\lambda>\sup _{i, U}\left|\lambda_{i}(U)\right| \quad(\lambda>2) .
$$

We hold such $\lambda$ fixed; hence $k$ is a function of $h$. Let $\theta=\left\{\theta_{n}\right\}$ be an equidistributed sequence in $(0,1)$. Let $m, n$ be integers such that $n \geq 0$ and $P_{m, n}=(2 m h, n k)$. We set

$$
A_{m, n}=\left(2\left(m+\theta_{n}\right) h, n k\right),
$$

which will be sampling points. We define a diamond shaped domain $\Delta_{m, n}$ by the vertices

$$
P_{m, n+1}, A_{m-1, n}, P_{m, n-1}, A_{m, n} .
$$

This domain is called the interaction diamond centered at $P_{m, n}$. A curve which consists of segments joining $P_{m, n}$ to $A_{m, n+1}$ or $A_{m, n-1}$, and $A_{m, n}$ to $P_{m+1, n+1}$ or 
$P_{m+1, n-1}$ is called an approximate space-like curve. We can partially order these approximate space-like curves: $I>J$ if every point of $J$ is either on $I$ or contained between $I$ and $t=0$. We denote by $O_{1}$ and $O_{2}$ the approximate space-like curves lying between $t=k$ and $t=0$.

We construct an approximate solution $U_{h, \theta}(x, t)$ as follows. At $t=0$, we solve the steady state equation (7) in the interval $2 m h<x<2(m+1) h$ with the initial value:

$$
U(2(m+\theta) h)=U_{0}\left(A_{m, 0}\right)
$$

and adopt $U(x)$ as the approximation in the interval $(2 m h, 2(m+1) h)$ at $t=0$. Suppose that the approximate solution is constructed for $0 \leq t<n k$, and the value $U_{h}(x, n k-0)$ exists. In the same manner as above, we solve the equation (7) in each interval $2 m h<x<2(m+1) h$ with the initial value

$$
U(2(m+\theta) h)=U_{h}(2(m+\theta) h, n k-0)
$$

and define

$$
U_{h}(x, n k)=U(x), \quad 2 m h<x<2(m+1) h .
$$

Next we solve the original hyperbolic system

$$
\frac{\partial}{\partial t} V+\frac{\partial}{\partial x} F(V)=G(x, V), \quad(x, t) \in(2(m-1) h, 2(m+1) h) \times(n k,(n+1) k)
$$

with the discontinuous initial data

$$
V(x, n k)=U_{h}(x, n k), \quad x \in(2(m-1) h, 2(m+1) h), x \neq m h .
$$

For simplicity, we consider $(2 m h, n k)$ as the origin and, denoting by $V_{L}(x), V_{R}(x)$ steady state solutions defined on $x<0, x>0$, respectively, we express the initial data (19) as

$$
V(x, 0)= \begin{cases}V_{L}(x), & x<0, \\ V_{R}(x), & x>0 .\end{cases}
$$

This problem was extensively studied by $\mathrm{Li}$ and $\mathrm{Yu}[10]$, and local solutions are obtained. However, the structures of their solutions are complicated and not suitable to the study of the interaction of waves. Hence we adopt approximate solutions introduced in Liu [12]. The following lemma is fundamental in the estimates of the approximate solution.

Lemma 3.1. Let $V(x), W(x)$ be steady state solutions. If $\left|\lambda_{p}(V(x))\right|,\left|\lambda_{p}(W(x))\right| \geq$ $c \alpha_{*}(c>0)$ and $V(x), W(x)$ are close to a constant vector $U_{0}$, then it follows that

$$
V\left(x_{2}\right)-W\left(x_{2}\right)=V\left(x_{1}\right)-W\left(x_{1}\right)+O(1) \frac{G\left|x_{2}-x_{1}\right|}{\alpha_{*}^{2}}\left|V\left(x_{1}\right)-W\left(x_{1}\right)\right| .
$$

Proof.

$$
\begin{aligned}
\frac{d}{d x}\{V(x)-W(x)\} & =\sum_{j=1}^{n}\left\{\frac{g_{j}(x, V(x))}{\lambda_{j}(V(x))} R_{j}(V(x))-\frac{g_{j}(x, W(x))}{\lambda_{j}(W(x))} R_{j}(W(x))\right\} \\
& =O(1) \frac{G}{\alpha_{*}^{2}}(V(x)-W(x)) .
\end{aligned}
$$


Hence we have

$$
V(x)-W(x)=\left(V\left(x_{1}\right)-W\left(x_{1}\right)\right) e^{O(1) G\left|x-x_{1}\right| / \alpha_{*}^{2}},
$$

which implies (21).

Suppose that the strength of the above discontinuity is $\ll \alpha_{*}$ (involving only weak waves). We construct the approximate solution by perturbing the solution to the Riemann problem for the system of conservation laws

$$
\frac{\partial}{\partial t} V+\frac{\partial}{\partial x} F(V)=0, \quad(x, t) \in R \times R_{+}
$$

with initial data

$$
V(x, 0)= \begin{cases}V_{L}(0), & x<0, \\ V_{R}(0), & x>0 .\end{cases}
$$

By Lax [7], a solution exists, and this solution consists of $(n+1)$ constant regions $V_{L}(0)=V_{0}, V_{1}, \ldots, V_{n}=V_{R}(0)$ connected by rarefaction waves and shock waves. Moreover, the solution of this form is unique provided the intermediate constant vectors are restricted to $\Omega$. Let $V_{j}(x), 0 \leq j \leq n$ denote the steady state solution with initial value

$$
V_{j}(0)=V_{j}
$$

The approximate solution of (1) with (25) consists of the steady states $V_{j}(x), 0 \leq j \leq n$ separated by approximate $j$-elementary waves.

If $\left\langle V_{j-1}, V_{j}\right\rangle$ is a shock wave with shock speed $\sigma_{j}=\sigma\left(V_{j-1}, V_{j}\right)$, we simply set

$$
V(x, t)= \begin{cases}V_{j-1}(x), & x<\sigma_{j} t \\ V_{j}(x), & x>\sigma_{j} t\end{cases}
$$

Then we have the following estimate.

Proposition 3.1. Assume that $V_{p-1}, V_{p} \notin \mathcal{N}_{p}$. Then

$$
\sigma_{j}\left[V_{j-1}\left(\sigma_{j} t\right)-V_{j}\left(\sigma_{j} t\right)\right]-\left[F\left(V_{j-1}\left(\sigma_{j} t\right)\right)-F\left(V_{j}\left(\sigma_{j} t\right)\right)\right]=O(1) \frac{\sigma_{j} G t}{\alpha_{*}}\left|V_{j-1}-V_{j}\right|
$$

for $1 \leq j \leq n$ where $G$ denote the maximum of the quantity $G(x)$ in (4).

Proof. We set $\sigma=\sigma_{j}$ and

$$
\Sigma(t)=\sigma\left[V_{j-1}(\sigma t)-V_{j}(\sigma t)\right]-\left[F\left(V_{j-1}(\sigma t)\right)-F\left(V_{j}(\sigma t)\right)\right] .
$$

Then differentiating $\Sigma(t)$, we find by (8) that

$$
\begin{aligned}
\frac{d \Sigma}{d t}= & \sigma^{2}\left\{\frac{d}{d x} V_{j-1}(x)-\frac{d}{d x} V_{j}(x)\right\}-\sigma\left\{\frac{d}{d x} F\left(V_{j-1}(x)\right)-\frac{d}{d x} F\left(V_{j}(x)\right)\right\} \\
= & \sigma^{2} \sum_{j=1}^{n}\left\{\frac{g_{j}\left(x, V_{j-1}(x)\right)}{\lambda_{j}\left(V_{j-1}(x)\right)} R_{j}\left(V_{j-1}(x)\right)-\frac{g_{j}\left(x, V_{j}(x)\right)}{\lambda_{j}\left(V_{j}(x)\right)} R_{j}\left(V_{j}(x)\right)\right\} \\
& -\sigma\left[G\left(x, V_{j-1}(x)\right)-G\left(x, V_{j}(x)\right)\right]
\end{aligned}
$$

$(x=\sigma t)$. Since $\sigma_{j}=O(1) \frac{1}{2}\left\{\lambda_{j}\left(V_{j-1}\right)+\lambda_{j}\left(V_{j}\right)\right\}$, we have

$$
\frac{d \Sigma}{d t}(t)=O(1) \frac{\sigma_{j} G}{\alpha_{*}}\left|V_{j-1}-V_{j}\right|
$$


and, hence, the proposition.

When $\left\langle V_{j-1}, V_{j}\right\rangle=\widehat{V}_{j}(x / t)$ is a rarefaction wave, we first define two $j$-characteristic curves which will be edges of the fan of characteristics

$$
\begin{aligned}
x_{j-1}(t): & \frac{d x}{d t}=\lambda_{j}\left(V_{j-1}(x)\right), & & x(0)=0 \\
x_{j}(t): & \frac{d x}{d t}=\lambda_{j}\left(V_{j}(x)\right), & & x(0)=0 .
\end{aligned}
$$

In the region $x_{j-1}(t)<x<x_{j}(t)$, we solve the singular boundary-value problem

$$
\begin{gathered}
\frac{\partial V}{\partial t}+F^{\prime}(V) \frac{\partial V}{\partial x}=G(x, V), \\
V\left(x_{j-1}(t), t\right)=V_{j-1}\left(x_{j-1}(t)\right), \\
\lim _{t \rightarrow 0} V(\eta t, t)=\widehat{V}_{j}(\eta)
\end{gathered}
$$

Since the solution $V(x, t)$ has a singularity at $t=0$, the local existence of the solution does not follow from the standard existence theorem, (for example, Courant [2]). However, Liu [12] showed that the solution can be obtained by integration along the characteristics with the data on the characteristic curve $x=x_{j-1}(t)$; details are found in [12]. The solution has a possible discontinuity along $x=x_{j}(t)$, which reflects the production of secondary shock waves (Glimm et. al. [5]). We have the estimate

Proposition 3.2. Assume that $\left|\lambda_{p}\left(V_{p-1}(x)\right)\right|,\left|\lambda_{p}\left(V_{p}(x)\right)\right| \geq c \alpha_{*}(c>0)$. Then

$$
\left|V\left(x_{j}(t), t\right)-V_{j}\left(x_{j}(t)\right)\right|=O(1) \frac{G t}{\alpha_{*}^{2}}\left|V_{j-1}-V_{j}\right| .
$$

Proof. We find, by the construction of the approximate solution in [12], that

$$
V\left(x_{j-1}(t), t\right)-V\left(x_{j}(t)-0, t\right)=V_{j-1}-V_{j}+O(1) \frac{G t}{\alpha_{*}^{2}}\left|V_{j-1}-V_{j}\right|
$$

Differentiating $V_{j-1}(x)$ and $V_{j}(x)$ along $x=x_{j}(t)$, we also have

$$
V_{j-1}\left(x_{j}(t)\right)-V_{j}\left(x_{j}(t)\right)=V_{j-1}-V_{j}+O(1) \frac{G t}{\alpha_{*}^{2}}\left|V_{j-1}-V_{j}\right|
$$

Hence we obtain

$$
V_{j}\left(x_{j}(t)\right)-V\left(x_{j}(t)-0, t\right)=V_{j-1}\left(x_{j}(t)\right)-V_{j-1}\left(x_{j}(t)\right)+O(1) \frac{G t}{\alpha_{*}^{2}}\left|V_{j-1}-V_{j}\right| .
$$

Since $x_{j}(t)-x_{j-1}(t)=O(1) t\left|V_{j-1}-V_{j}\right|$, we have the proposition.

In this way we have constructed the approximate solution when only weak waves are involved.

Next we treat the case where single strong shock wave is involved. Here we apply the front tracking scheme introduced by Chern [1], which is to trace the location of the single strong shock wave. Suppose that the approximate solution is constructed by front tracking for $0 \leq t<n k$, and the value $U_{h}\left(x, n k-0\right.$ ) exists (we take $U_{0}(x)=$ $U_{h}(x, n k-0)$ at $\left.t=0\right)$. We denote by $x=x_{\mathcal{F}}(t)$ the front of the single strong $p$-shock wave and

$$
m_{\mathcal{F}}(n)=\left[\frac{x_{\mathcal{F}}(n k)}{2 h}\right]
$$


where $[a]$ is Gauss' symbol; by abuse of notation $m_{\mathcal{F}}=m_{\mathcal{F}}(n)$ and $x_{\mathcal{F}}=x_{\mathcal{F}}(t)$. We call the interval $\left(2\left(m_{\mathcal{F}}-1\right) h, 2\left(m_{\mathcal{F}}+2\right) h\right)$ the "front region" at $t=n k$. In the front region, we solve the steady state equation (7) in the interval $2\left(m_{\mathcal{F}}-1\right) h<x<x_{\mathcal{F}}$ and $x_{\mathcal{F}}<x<2\left(m_{\mathcal{F}}+2\right) h$ with the initial value

$$
\begin{aligned}
& U\left(2\left(m_{\mathcal{F}}-1+\theta\right) h\right)=U_{h}\left(A_{m_{\mathcal{F}}-1, n}\right), \\
& U\left(2\left(m_{\mathcal{F}}+1+\theta\right) h\right)=U_{h}\left(A_{m_{\mathcal{F}}+1, n}\right),
\end{aligned}
$$

respectively, and denote these solutions by $U^{-}$and $U^{+}$, respectively. Next we solve the Riemann problem for the system of conservation laws

$$
\frac{\partial}{\partial t} U+\frac{\partial}{\partial x} F(U)=0, \quad(x, t) \in R \times R_{+}
$$

with initial data

$$
U(x, n k)= \begin{cases}U^{-}\left(x_{\mathcal{F}}(n k)\right), & x<x_{\mathcal{F}}(n k), \\ U^{+}\left(x_{\mathcal{F}}(n k)\right), & x>x_{\mathcal{F}}(n k) .\end{cases}
$$

The solution $U(x, t)$ contains a relatively strong $p$-shock wave which separates the $p-1$-constant region $U_{p-1}$ and the $p$-constant region $U_{p}$. We solve again the steady state equation $(7)$ in the interval $2\left(m_{\mathcal{F}}-1\right) h<x<x_{\mathcal{F}}$ and $x_{\mathcal{F}}<x<2\left(m_{\mathcal{F}}+2\right) h$ with the initial value

$$
\begin{aligned}
& U\left(x_{\mathcal{F}}(n k)\right)=U_{p-1}, \\
& U\left(x_{\mathcal{F}}(n k)\right)=U_{p},
\end{aligned}
$$

respectively, and denote these solutions by $U_{m_{\mathcal{F}}-1}$ and $U_{m_{\mathcal{F}}+1}$, respectively, which will be the approximation in the front region at $t=n k$. For $n k \leq t<(n+1) k$, we continue the front to be

$$
x_{\mathcal{F}}(t): \quad \sigma_{p}(t-n k)+x_{\mathcal{F}}(n k)
$$

and define the approximate solution $U_{h}$ by

$$
U_{h}(x, t)= \begin{cases}U_{m_{\mathcal{F}}-1}(x), & \left(2 m_{\mathcal{F}}-1\right) h<x<x_{\mathcal{F}}(t), \\ U_{m_{\mathcal{F}}+1}(x), & x_{\mathcal{F}}(t)<x<\left(2 m_{\mathcal{F}}+3\right) h .\end{cases}
$$

Although the discontinuities at $x=2\left(m_{\mathcal{F}}-1\right) h$ and $2\left(m_{\mathcal{F}}+2\right) h$ (the end points of the front region) are weak, a special construction is needed: in (25), we have to replace $V_{R}(0)=U_{m_{\mathcal{F}}-1}\left(2\left(m_{\mathcal{F}}-1\right) h\right)$ and $V_{L}(0)=U_{m_{\mathcal{F}}+1}\left(2\left(m_{\mathcal{F}}+2\right) h\right)$, respectively.

\section{Local interaction estimates}

We first study the case involving only weak waves. Let $\gamma$ denote the set of waves issuing from $(2 m h,(n+1) k)$. Our construction of approximate solutions shows that if $0<\theta_{n} \leq$ $\frac{1}{2}$, then $\gamma$ depends on the waves issuing from $(2(m-1) h, n k)$ and $(2 m h, n k)$, while if $\frac{1}{2}<\theta_{n}<1$, then it depends on the waves issuing from $(2 m h, n k)$ and $(2(m+1) h, n k)$. Now suppose that $0<\theta_{n} \leq \frac{1}{2}$. We denote by $\alpha$ the set of waves issuing from $(2 m h, n k)$ and by $\beta=\beta^{R}$ the set of waves issuing from $(2(m-1) h, n k)$ and entering the interaction diamond $\Delta_{m, n}$. We may assume that $\beta_{j}=0$ for $j<p$. Let $U_{L}(x)$ and $U_{R}(x)$ be steady state solutions connected by $\gamma$ and $U_{M}(x)$ another steady state 
solution between $\beta^{R}$ and $\alpha$. The local interaction estimates in this case consist in comparing the solution to the hyperbolic system (1) with initial data

$$
U(x, 0)= \begin{cases}U_{L}(x), & x<2 m h, \\ U_{R}(x), & x>2 m h\end{cases}
$$

and the solution with initial data

$$
U(x, 0)= \begin{cases}U_{L}(x), & x<2(m-1) h \\ U_{M}(x), & 2(m-1)<x<2 m h \\ U_{R}(x), & x>2 m h\end{cases}
$$

We set

$$
\begin{array}{rlrl}
V_{-} & \equiv U_{L}(2(m-1) h), & V_{+} & \equiv U_{M}(2(m-1) h), \\
W_{-} \equiv U_{M}(2 m h), & W_{+} \equiv U_{R}(2 m h), \\
U_{-} \equiv U_{L}(2 m h), & U_{+} \equiv W_{+} \equiv U_{R}(2 m h) .
\end{array}
$$

Denote by $\left\langle U_{-}, U_{+}\right\rangle_{j}$ the magnitude of the $j$-wave in the solution to the Riemann problem $(G(x, U) \equiv 0)$ with $U_{L}=U_{-}$and $U_{R}=U_{+}$, and set

$$
\gamma_{j}=\left\langle U_{-}, U_{+}\right\rangle_{j}, \beta_{j}=\left\langle V_{-}, V_{+}\right\rangle_{j}, \alpha_{j}=\left\langle W_{-}, W_{+}\right\rangle_{j}, \quad 1 \leq j \leq n .
$$

We say that $\beta_{i}$ and $\alpha_{j}$ are "approaching" if either one of the following holds: (i) $i>j$, or (ii) $i=j$ and at least one of them is a shock. We define

$$
Q_{0}\left(\Delta_{m, n}\right)=Q_{0}\left(\beta^{R}, \alpha\right)=\sum\left\{\left|\beta_{i} \alpha_{j}\right|: \beta_{i} \text { and } \alpha_{j} \text { are approaching }\right\} .
$$

If $V_{+}=W_{-}$, then $Q_{0}\left(\beta^{R}, \alpha\right)$ is denoted by $Q_{0}\left(U_{-}, W_{-}, U_{+}\right)$. We set

$$
\widehat{Q}\left(\Delta_{m, n}\right)=Q_{0}\left(\beta^{R}, \alpha\right)+\frac{G_{m} h}{\alpha_{*}^{2}}\left|\beta^{R}\right|
$$

where $G_{m}$ is the maximum of $|G(x, U)|+\left|G_{U}^{\prime}(x, U)\right|$ for $2(m-1)<x<(2 m+1) h$ and $U$ in a small neighborhood of $V_{ \pm}$and $W_{ \pm}$. Our local interaction estimates are the following.

Lemma 4.1 (Liu [12]). Assume that $\left|\lambda_{p}\left(V_{p-1}(x)\right)\right|,\left|\lambda_{p}\left(V_{p}(x)\right)\right| \geq c \alpha_{*}(c>0)$ and $U_{L}(x), U_{M}(x)$, and $U_{R}(x)$ are close to a constant vector $U_{0}$. Then

$$
\gamma_{j}=\beta_{j}+\alpha_{j}+O(1) \widehat{Q}\left(\Delta_{m, n}\right), \quad 1 \leq j \leq n .
$$

Here $O(1)$ depends only on $U_{L}, U_{R}$, and the system.

Proof. It follows from Lemma 3.1 that

$$
\begin{aligned}
& W_{-}-U_{-}=V_{+}-V_{-}+O(1) \frac{G_{m} h}{\alpha_{*}^{2}}\left|V_{+}-V_{-}\right|, \\
& U_{+}-W_{-}=W_{+}-W_{-} .
\end{aligned}
$$

Since the constant states composing the solution to the Riemann problem are differentiable with respect to the initial data, we have

$$
\begin{aligned}
& \left\langle U_{-}, W_{-}\right\rangle_{j}=\beta_{j}+O(1) \frac{G_{m} h}{\alpha_{*}^{2}}\left|V_{+}-V_{-}\right|, \\
& \left\langle W_{-}, U_{+}\right\rangle_{j}=\left\langle W_{-}, W_{+}\right\rangle_{j}=\alpha_{j} .
\end{aligned}
$$


Hence,

$$
\begin{aligned}
\gamma_{j} & =\left\langle U_{-}, U_{+}\right\rangle_{j} \\
& =\left\langle U_{-}, W_{-}\right\rangle_{j}+\left\langle W_{-}, U_{+}\right\rangle_{j}+O(1) Q_{0}\left(U_{-}, W_{-}, U_{+}\right) \\
& =\beta_{j}+\alpha_{j}+O(1) Q_{0}\left(U_{-}, W_{-}, U_{+}\right)+O(1) \frac{G_{m} h}{\alpha_{*}^{2}}\left|V_{+}-V_{-}\right| \\
& =\beta_{j}+\alpha_{j}+O(1) Q_{0}\left(\beta^{R}, \alpha\right)+O(1) \frac{G_{m} h}{\alpha_{*}^{2}}\left|\beta^{R}\right| .
\end{aligned}
$$

Thus, the lemma follows.

Next we study the case involving the single strong shock wave. We set $m_{\mathcal{F}}(n)=m$ and denote by $\beta_{*}=\left(0, \ldots, 0, \beta_{p}, 0, \ldots, 0\right)$ the strong $p$-shock wave whose speed is $\sigma$. Assume as before that $0<\theta_{n} \leq \frac{1}{2}$. We denote by $\alpha$ the set of waves issuing from $(2(m-1) h, n k)$. The waves in $\alpha$ entering the interaction diamond $\Delta_{m, n}$ are denoted by $\alpha^{R}$, and the waves entering $\Delta_{m-1, n}$ by $\alpha^{L}$. We denote by $\gamma^{R}$ the set of waves issuing from $(2(m-2) h, n k)$ and entering the interaction diamond $\Delta_{m-1, n}$. Let $U_{L}(x), U_{M}(x)$, and $U_{R}(x)$ be steady state solutions such that $\alpha^{R}$ connects $U_{L}(x)$ and $U_{M}(x)$, and $\beta$ connects $U_{M}(x)$ and $U_{R}(x)$.

As before, we set

$$
\begin{aligned}
V_{-} & \equiv U_{L}(2(m-1) h), & V_{+} & \equiv U_{M}(2(m-1) h) \\
W_{-} & \equiv U_{M}\left(x_{\mathcal{F}}(n)\right), & W_{+} & \equiv U_{R}\left(x_{\mathcal{F}}(n)\right) .
\end{aligned}
$$

Also

$$
\begin{aligned}
\widetilde{W}_{-} \equiv U_{M}\left(x_{\mathcal{F}}(n+1)\right), & \widetilde{W}_{+} & \equiv U_{R}\left(x_{\mathcal{F}}(n+1)\right), \\
U_{-} \equiv U_{L}\left(x_{\mathcal{F}}(n+1)\right), & U_{+} & \equiv \widetilde{W}_{+} \equiv U_{R}\left(x_{\mathcal{F}}(n+1)\right) .
\end{aligned}
$$

Let $\sigma$ denote the speed of the single strong shock wave issuing from $\left(x_{\mathcal{F}}, n k\right)$ and $U_{R}(t)=U_{R}\left(\sigma(t-n k)+x_{\mathcal{F}}(n)\right), U_{M}(t)=U_{M}\left(\sigma(t-n k)+x_{\mathcal{F}}(n)\right)$. We find that

$$
\begin{aligned}
\frac{d}{d t}\left\{U_{R}(t)-U_{M}(t)\right\} & =\sigma\left\{\frac{d}{d x} U_{R}(x)-\frac{d}{d x} U_{M}(x)\right\} \\
& =\sigma \sum_{j=1}^{n}\left\{\frac{g_{j}\left(x, U_{R}(x)\right)}{\lambda_{j}\left(U_{R}(x)\right)} R_{j}\left(U_{R}(x)\right)-\frac{g_{j}\left(x, U_{M}(x)\right)}{\lambda_{j}\left(U_{M}(x)\right)} R_{j}\left(U_{M}(x)\right)\right\} \\
& =O(1) \frac{\sigma G_{m}}{\alpha_{*}} .
\end{aligned}
$$

Hence, integrating from $n k$ to $(n+1) k$, we have:

Proposition 4.1. If $\beta_{*}=O(1) \alpha_{*}$, then

$$
\widetilde{W}_{+}-\widetilde{W}_{-}=W_{+}-W_{-}+O(1) \frac{\sigma G_{m} h}{\alpha_{*}} .
$$

Now we carry out the interaction estimates involving the single strong shock wave. From Lemma 3.1,

$$
\widetilde{W}_{-}-U_{-}=V_{+}-V_{-}+O(1) \frac{G_{m} h}{\alpha_{*}^{2}}\left|V_{+}-V_{-}\right|
$$


The above proposition says that

$$
\begin{aligned}
U_{+}-\widetilde{W}_{-} & =\widetilde{W}_{+}-\widetilde{W}_{-} \\
& =W_{+}-W_{-}+O(1) \frac{\sigma G_{m} h}{\alpha_{*}} .
\end{aligned}
$$

Hence it follows from the continuity of the Riemann solver with respect to the data that

$$
\begin{aligned}
& \left\langle U_{-}, \widetilde{W}_{-}\right\rangle_{j}=\alpha_{j}^{R}+O(1) \frac{G_{m} h}{\alpha_{*}^{2}}\left|\alpha^{R}\right|, \\
& \left\langle\widetilde{W}_{-}, U_{+}\right\rangle_{j}=\beta_{j}+O(1) \frac{\sigma G_{m} h}{\alpha_{*}},
\end{aligned}
$$

where $\beta_{p}=\beta_{*}$ and $\beta_{j}=0(j \neq p)$. Hence, denoting $\delta_{j}=\left\langle U_{-}, U_{+}\right\rangle_{j}$, we have

$$
\begin{aligned}
\delta_{j}= & \alpha_{j}^{R}+\beta_{j}+O(1) Q_{0}\left(\alpha^{R}, \beta_{*}\right)+O(1) \frac{G_{m} h}{\alpha_{*}^{2}}\left|V_{+}-V_{-}\right| \\
= & \begin{cases}\alpha_{j}^{R}+O(1) \frac{G_{m} h}{\alpha_{*}^{2}}\left|\alpha^{R}\right|+O(1) \frac{\sigma G_{m} h}{\alpha_{*}}+O(1) \beta_{*} \sum_{l \geq p}\left|\alpha_{l}^{R}\right|, & j \neq p, \\
\beta_{*}+\alpha_{p}^{R}+O(1) \frac{G_{m} h}{\alpha_{*}^{2}}\left|\alpha^{R}\right|+O(1) \frac{\sigma G_{m} h}{\alpha_{*}}+O(1) \beta_{*} \sum_{l \geq p}\left|\alpha_{l}^{R}\right|, & j=p .\end{cases}
\end{aligned}
$$

Here the $p$-th wave $\delta_{p}$ is the strong shock wave leaving $\Delta_{m-1, n}$, which is denoted from now on by $\beta_{*}^{\prime}$. Let $U_{p-1}$ and $U_{p}$ denote constant states connected by $\delta_{p}$. We denote by $\widetilde{U}_{M}(x)$ the steady state solution satisfying

$$
\widetilde{U}_{M}\left(x_{\mathcal{F}}((n+1) k)\right)=U_{p-1}
$$

and set

$$
\widetilde{V}_{+}=\widetilde{U}_{M}(2(m-1) h)
$$

Then

$$
\widetilde{V}_{+}-V_{-}=U_{p-1}-U_{-}+O(1) \frac{G_{m} h}{\alpha_{*}^{2}}\left|U_{p-1}-U_{-}\right|
$$

Hence

$$
\left\langle V_{-}, \tilde{V}_{+}\right\rangle_{j}= \begin{cases}\alpha_{j}^{R}+O(1) \frac{G_{m} h}{\alpha_{*}^{2}}\left|\alpha^{R}\right|+O(1) \frac{\sigma G_{m} h}{\alpha_{*}}+O(1) \beta_{*} \sum_{l \geq p}\left|\alpha_{l}^{R}\right|, & 1 \leq j<p, \\ O(1) \frac{G_{m} h}{\alpha_{*}^{2}}\left|\alpha^{R}\right|+O(1) \frac{\sigma G_{m} h}{\alpha_{*}}+O(1) \beta_{*} \sum_{l \geq p}\left|\alpha_{l}^{R}\right|, & p \leq j \leq n .\end{cases}
$$

The interaction of $\alpha^{L}$ and $\left\langle V_{-}, \widetilde{V}_{+}\right\rangle$produces $\tilde{\alpha}$ such that

$$
\widetilde{\alpha}_{j}=\left\{\begin{array}{l}
\alpha_{j}+O(1) \frac{G_{m} h}{\alpha_{*}^{2}}\left|\alpha^{R}\right|+O(1) \frac{\sigma G_{m} h}{\alpha_{*}}+O(1) \beta_{*} \sum_{l \geq p}\left|\alpha_{l}^{R}\right|, \quad 1 \leq j<p, \\
\alpha_{j}^{L}+O(1) \frac{G_{m} h}{\alpha_{*}^{2}}\left|\alpha^{R}\right|+O(1) \frac{\sigma G_{m} h}{\alpha_{*}}+O(1) \beta_{*} \sum_{l \geq p}\left|\alpha_{l}^{R}\right|, \quad p \leq j \leq n .
\end{array}\right.
$$


We define the quantity $Q_{1}\left(\Delta_{m, n}\right)=Q_{1}\left(\alpha, \gamma ; \beta_{*}\right)$ by

$$
Q_{1}\left(\alpha, \gamma ; \beta_{*}\right)=\left|\beta_{*}\right| \sum_{l \geq p}\left|\alpha_{l}^{R}\right|+\sum\left\{\left|\alpha_{l} \gamma_{m}^{R}\right|: \alpha_{l} \text { and } \gamma_{m}^{R}\right. \text { are approaching, }
$$

$l$ satisfying $1 \leq l<p$ or $\left.l \geq p, \alpha_{l}^{L} \neq 0\right\}$

Let $\epsilon$ denote the set of waves issuing from $\left(2\left(m_{\mathcal{F}}-1\right),(n+1) k\right)$ and leaving $\Delta_{m, n}$. Combining the above estimates, we find that $\epsilon$ has the estimates

$$
\begin{aligned}
& \epsilon_{j}=\left\{\begin{aligned}
\alpha_{j}+\gamma_{j}^{R}+ & O(1) \frac{G_{m} h}{\alpha_{*}^{2}}\left(\left|\alpha^{R}\right|+\left|\gamma^{R}\right|\right) \\
& +O(1) \frac{\sigma G_{m} h}{\alpha_{*}}+O(1) Q_{1}\left(\alpha, \gamma ; \beta_{*}\right), \quad 1 \leq j<p, \\
\alpha_{j}^{L}+\gamma_{j}^{R} & +O(1) \frac{G_{m} h}{\alpha_{*}^{2}}\left(\left|\alpha^{R}\right|+\left|\gamma^{R}\right|\right) \\
& +O(1) \frac{\sigma G_{m} h}{\alpha_{*}}+O(1) Q_{1}\left(\alpha, \gamma ; \beta_{*}\right), \quad p \leq j \leq n,
\end{aligned}\right. \\
& \beta_{*}^{\prime}=\beta_{*}+\alpha_{p}^{R}+O(1) \frac{G_{m} h}{\alpha_{*}^{2}}\left|\alpha^{R}\right|+O(1) \frac{\sigma G_{m} h}{\alpha_{*}}+O(1) Q_{1}\left(\alpha, \gamma ; \beta_{*}\right) .
\end{aligned}
$$

We set, as before,

$$
\widehat{Q}\left(\Delta_{m, n}\right)=Q_{1}\left(\Delta_{m, n}\right)+\frac{G_{m} h}{\alpha_{*}^{2}}\left(\left|\alpha^{R}\right|+\left|\gamma^{R}\right|\right)
$$

where $G_{m}$ is the maximum of $|G(x, U)|+\left|G_{U}^{\prime}(x, U)\right|$ for $2\left(m_{\mathcal{F}}-1\right)<x<\left(2 m_{\mathcal{F}}+1\right) h$ and $U$ in a small neighborhood of $V_{ \pm}$and $W_{ \pm}$. Then we have the local interaction estimates involving the single strong shock wave in the following form.

Lemma 4.2 (Chern [1]). Assume that $\left|\lambda_{p}\left(V_{p-1}(x)\right)\right|,\left|\lambda_{p}\left(V_{p}(x)\right)\right| \geq c \alpha_{*}(c>0)$, and $U_{L}(x), U_{M}(x)$, and $U_{R}(x)$ are close to a constant vector $U_{0}$. Then it follows that

$$
\begin{gathered}
\epsilon_{j}=\left\{\begin{array}{l}
\alpha_{j}+\gamma_{j}^{R}+O(1) \widehat{Q}\left(\Delta_{m, n}\right)+O(1) \frac{\sigma G_{m} h}{\alpha_{*}}, 1 \leq j<p, \\
\alpha_{j}^{L}+\gamma_{j}^{R}+O(1) \widehat{Q}\left(\Delta_{m, n}\right)+O(1) \frac{\sigma G_{m} h}{\alpha_{*}}, p \leq j \leq n,
\end{array}\right. \\
\beta_{*}^{\prime}=\beta_{*}+\alpha_{p}^{R}+O(1) \widehat{Q}\left(\Delta_{m, n}\right)+O(1) \frac{\sigma G_{m} h}{\alpha_{*}} .
\end{gathered}
$$

Here $O(1)$ depends only on $U_{L}, U_{R}$, and the system. 


\section{Global interaction estimates}

The restriction of the approximate solution $U_{h, \theta}$ to an approximate space-like curve $J$ consists of steady state regions, rarefaction waves, and shock waves. Let $\alpha=$ $\left(\alpha_{1}, \alpha_{2}, \ldots, \alpha_{n}\right), \beta=\left(\beta_{1}, \beta_{2}, \ldots, \beta_{n}\right)$ be two waves in $U_{h, \theta}$ crossing $J$. Suppose that $\alpha$ lies to the left of $\beta$ on $J$. We say that $\alpha_{i}$ and $\beta_{j}$ are "approaching" if either one of the following holds: (i) $i>j$, or (ii) $i=j$ and at least one of them is a shock. Let $W(J)$ denote the collection of the weak waves crossing $J$. Also $W_{a}(J)$ denotes the subset of $W(J)$ the elements of which are approaching the single strong shock wave. Let us define

$$
\begin{aligned}
L(J) & =\sum_{\alpha \in W(J)}|\alpha| \\
\|G\|_{1} & =\int_{-\infty}^{\infty} G(x) d x
\end{aligned}
$$

and let $\beta_{*}$ denote also the magnitude of the single strong shock wave crossing $J . L(J)$ together with $\beta_{*}$ measures the total variation of $U_{h, \theta}(x, t)$ on $J$.

The global interaction estimates show that the total amount of interaction in the Glimm approximate solutions is uniformly bounded. Lemma 4.2 indicates that the total amount of the secondary wave produced by the transonic waves is

$$
\sum_{n \geq 0} \frac{\left|\sigma_{n}\right| G_{m(n)} h}{\alpha_{*}}
$$

where the single strong shock wave enters $\Delta_{m(n), n}$ with speed $\sigma_{n}$.

Theorem 5.1. Assume that $\alpha_{*}$ and $\|G\|_{1} / \alpha_{*}^{2}$ are sufficiently small and $L(O) \ll \alpha_{*}$.

1. If we further assume that the quantity (55) is also small, then we have

$$
\sum_{\Delta} \widehat{Q}(\Delta) \leq O(1)\left\{L(O)+\alpha_{*}+\frac{\|G\|_{1}}{\alpha_{*}^{2}}\right\}^{2}+O(1) \sum_{n \geq 0} \frac{\left|\sigma_{n}\right| G_{m(n)} h}{\alpha_{*}}
$$

2. If we assume instead that the sign of the speed of the single strong shock front in the Glimm approximations never changes, then the following stronger estimate holds

$$
\sum_{\Delta} \widehat{Q}(\Delta)+\sum_{n \geq 0} \frac{\left|\sigma_{n}\right| G_{m(n)} h}{\alpha_{*}} \leq O(1)\left\{L(O)+\alpha_{*}+\frac{\|G\|_{1}}{\alpha_{*}^{2}}\right\}^{2} .
$$

This theorem is proved by Lemmas 5.1 and 5.2. We introduce the potential function $Q(J)$ defined for any $J$ by

$$
Q(J)=Q_{0}(J)+Q_{p}(J)+\sum_{j \neq p} Q_{j}(J)
$$


where

$$
\begin{aligned}
& Q_{0}(J)=\sum\{|\alpha \beta|: \alpha, \beta \in W(J) \text { and approaching }\}+\beta_{*} \sum\left\{|\alpha|: \alpha \in W_{a}(J)\right\},
\end{aligned}
$$

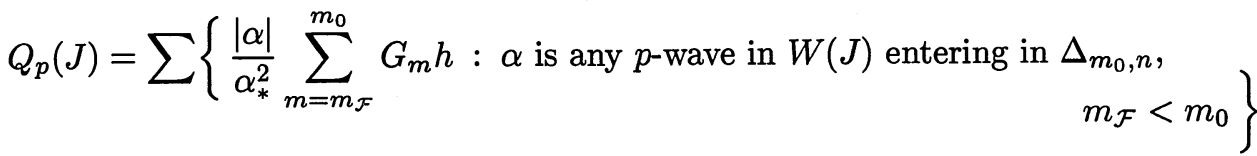

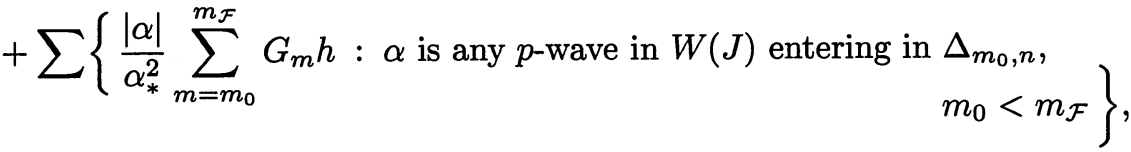

$$
\begin{aligned}
& Q_{j}(J)=\left\{\begin{array}{rr}
\sum\left\{\frac{|\alpha|}{\alpha_{*}^{2}} \sum_{m \leq m_{0}} G_{m} h: \alpha \text { is any } j \text {-wave in } W(J) \text { entering in } \Delta_{m_{0}, n}\right\} \\
1 \leq j<p, \\
\sum\left\{\frac{|\alpha|}{\alpha_{*}^{2}} \sum_{m \geq m_{0}} G_{m} h: \alpha \text { is any } j \text {-wave in } W(J) \text { entering in } \Delta_{m_{0}, n}\right\}, \\
p<j \leq n .
\end{array}\right.
\end{aligned}
$$

In defining $Q_{p}(J)$, we may assume that $\lambda_{p}>0$ in the left region of the strong shock wave and $\lambda_{p}<0$ in the right region, because of (13). We also note that

$$
\sum_{m \geq m_{0}} G_{m} h=O(1) \int_{m_{0} h}^{\infty} G(x) d x .
$$

Now suppose that $0<\theta_{n} \leq \frac{1}{2}$. As in the previous section, we denote by $\alpha$ the set of waves issuing from $\left(2 m_{0} h, n k\right)$ and by $\beta^{R}$ the set of waves issuing from $\left(2\left(m_{0}-1\right) h, n k\right)$ and entering the interaction diamond $\Delta$. The following lemma is fundamental in this section.

Lemma 5.1. Let $J_{1}, J_{2}$ be two consecutive approximate space-like curves such that $J_{2}>J_{1}$ and $\Delta$ is the diamond between them. Assume that $L\left(J_{1}\right), \beta_{*}$ and $\|G\|_{1} / \alpha_{*}^{2}$ are sufficiently small. Then we have

$$
\widehat{Q}(\Delta) \leq 2\left(Q\left(J_{1}\right)-Q\left(J_{2}\right)\right)+\frac{2|\sigma| G_{m_{0}} h}{\alpha_{*}} .
$$

Proof. Case 1. The single strong shock wave does not enter $\Delta$. Suppose that $\alpha$ and $\beta$ enter $\Delta$ and $\gamma$ leaves.

Estimates of $Q_{0}(J)$. The Glimm interaction estimate implies for large $K$ that

$$
Q_{0}\left(J_{2}\right) \leq Q_{0}\left(J_{1}\right)-\left(1-K L\left(J_{1}\right)\right) Q_{0}(\Delta)+K L\left(J_{1}\right) \frac{G_{m_{0}} h}{\alpha_{*}^{2}}\left|\beta^{R}\right|
$$

Estimates of $Q_{p}(J)$. If $m_{0}<m_{\mathcal{F}}$, we find that

$$
\frac{\left|\gamma_{p}\right|}{\alpha_{*}^{2}} \sum_{m_{1} \leq m \leq m_{\mathcal{F}}} G_{m} h \leq \frac{\left|\alpha_{p}\right|}{\alpha_{*}^{2}} \sum_{m_{1} \leq m \leq m_{\mathcal{F}}} G_{m} h+\frac{\left|\beta_{p}\right|}{\alpha_{*}^{2}} \sum_{m_{1} \leq m^{\prime} \leq m_{\mathcal{F}}} G_{m} h+\frac{K\|G\|_{1}}{\alpha_{*}^{2}} \widehat{Q}(\Delta)
$$


$\left(m_{1}=m_{0}\right.$ or $\left.m_{0}+1\right)$. If $m_{0}>m_{\mathcal{F}}$, we have in the same manner

$$
\frac{\left|\gamma_{p}\right|}{\alpha_{*}^{2}} \sum_{m_{\mathcal{F}} \leq m \leq m_{0}} G_{m} h \leq \frac{\left|\alpha_{p}\right|}{\alpha_{*}^{2}} \sum_{m_{\mathcal{F}} \leq m \leq m_{1}} G_{m} h+\frac{K\|G\|_{1}}{\alpha_{*}^{2}} \widehat{Q}(\Delta),
$$

$\left(m_{1}=m_{0}\right.$ or $\left.m_{0}-1\right)$. Hence

$$
Q_{p}\left(J_{2}\right) \leq Q_{p}\left(J_{1}\right)-\frac{\left|\beta_{p}\right|}{\alpha_{*}^{2}} G_{m_{0}} h+\frac{K\|G\|_{1}}{\alpha_{*}^{2}} \widehat{Q}(\Delta) .
$$

Estimates of $Q_{j}(J), j \neq p$. For $j>p$, we have

$$
\frac{\left|\gamma_{j}\right|}{\alpha_{*}^{2}} \sum_{m \geq m_{1}} G_{m} h \leq \frac{\left|\alpha_{j}\right|}{\alpha_{*}^{2}} \sum_{m \geq m_{1}} G_{m} h+\frac{\left|\beta_{j}\right|}{\alpha_{*}^{2}} \sum_{m \geq m_{1}} G_{m} h+\frac{K\|G\|_{1}}{\alpha_{*}^{2}} \widehat{Q}(\Delta)
$$

$\left(m_{1}=m_{0}\right.$ or $\left.m_{0}-1\right)$. For $j<p$, similarly,

$$
\frac{\left|\gamma_{j}\right|}{\alpha_{*}^{2}} \sum_{m \leq m_{1}} G_{m} h \leq \frac{\left|\alpha_{p}\right|}{\alpha_{*}^{2}} \sum_{m \leq m_{1}} G_{m} h+\frac{K\|G\|_{1}}{\alpha_{*}^{2}} \widehat{Q}(\Delta)
$$

$\left(m_{1}=m_{0}\right.$ or $\left.m_{0}-1\right)$. Hence

$$
Q_{j}\left(J_{2}\right) \leq Q_{j}\left(J_{1}\right)-\frac{\left|\beta_{j}\right|}{\alpha_{*}^{2}} G_{m_{0}} h+\frac{K\|G\|_{1}}{\alpha_{*}^{2}} \widehat{Q}(\Delta) .
$$

Then it follows from (61) and (62) that

$$
\begin{aligned}
\sum_{j=1}^{n} Q_{j}\left(J_{2}\right) \leq \sum_{j=1}^{n} Q_{j}\left(J_{1}\right)+\frac{K\|G\|_{1}}{\alpha_{*}^{2}} \widetilde{Q}(\Delta) \\
-\left\{1-K L\left(J_{1}\right)-\frac{K\|G\|_{1}}{\alpha_{*}^{2}}\right\} \frac{G_{m_{0}} h}{\alpha_{*}^{2}}\left|\beta^{R}\right| .
\end{aligned}
$$

Thus combining (60) and the above estimates, we obtain

$$
\begin{aligned}
Q\left(J_{2}\right) & \leq Q\left(J_{1}\right)-\left\{1-K L\left(J_{1}\right)-\frac{K\|G\|_{1}}{\alpha_{*}^{2}}\right\}\left\{Q_{0}(\Delta)+\frac{G_{m_{0}} h}{\alpha_{*}^{2}}\left|\beta^{R}\right|\right\} \\
& \leq Q\left(J_{1}\right)-\left\{1-K L\left(J_{1}\right)-\frac{K\|G\|_{1}}{\alpha_{*}^{2}}\right\} \widehat{Q}(\Delta) .
\end{aligned}
$$

Case 2. The single strong shock wave enters $\Delta$. Let $\Delta$ denote the interaction diamond between $J_{2}$ and $J_{1}$. We adopt the notations in the proof of Lemma 4.2 except that we use $m_{\mathcal{F}}(n)=m_{0}$.

Estimates of $Q_{0}(J)$. It follows from (53) and the Glimm interaction estimates that

$\sum\left\{|\alpha \beta|: \alpha, \beta \in W\left(J_{2}\right)\right.$ and approaching $\}$

$$
\begin{aligned}
& \leq \sum\left\{|\alpha \beta|: \alpha, \beta \in W\left(J_{1}\right) \text { and approaching }\right\} \\
& -\sum\left\{\left|\alpha_{l} \gamma_{m}^{R}\right|: \alpha_{l} \text { and } \gamma_{m}^{R}\right. \text { approaching, } \\
& \left.\quad l \text { satisfying } 1 \leq l<p \text { or } l \geq p, \alpha_{l}^{L} \neq 0\right\} \\
& +K L\left(J_{1}\right) \frac{G_{m_{0}} h}{\alpha_{*}^{2}}\left(\left|\alpha^{R}\right|+\left|\gamma^{R}\right|\right)+K L\left(J_{1}\right) \frac{|\sigma| G_{m_{0}} h}{\alpha_{*}} .
\end{aligned}
$$


Obviously,

$$
\begin{aligned}
\beta_{*}^{\prime} \sum\{|\alpha|: \alpha & \left.\in W_{a}\left(J_{2}\right)\right\} \leq \beta_{*} \sum_{l \geq p}\left|\alpha_{l}^{L}\right|+\beta_{*} \sum_{l \geq p}\left|\gamma_{l}^{R}\right| \\
& +\beta_{*} \sum\left\{|\alpha|: \alpha \in W_{a}\left(J_{1}\right), \text { not entering } \Delta\right\} \\
& +K \beta_{*} \frac{G_{m_{0}} h}{\alpha_{*}^{2}}\left(\left|\alpha^{R}\right|+\left|\gamma^{R}\right|\right)+K \beta_{*} \frac{|\sigma| G_{m} h}{\alpha_{*}}+K \beta_{*} Q_{1}(\Delta) \\
\leq \beta_{*} & \sum\left\{|\alpha|: \alpha \in W_{a}\left(J_{1}\right)\right\} \\
& -\beta_{*} \sum_{l \geq p}\left|\alpha_{l}^{R}\right|+K \beta_{*} \frac{G_{m_{0}} h}{\alpha_{*}^{2}}\left(\left|\alpha^{R}\right|+\left|\gamma^{R}\right|\right)+K \beta_{*} \frac{|\sigma| G_{m_{0}} h}{\alpha_{*}} .
\end{aligned}
$$

Here we may assume $|\alpha| \ll \beta_{*}$. Hence, we have

$$
\begin{aligned}
Q_{0}\left(J_{2}\right) \leq & Q_{0}\left(J_{1}\right)-\left(1-K L\left(J_{1}\right)-K \beta_{*}\right) Q_{1}(\Delta) \\
& +K\left(L\left(J_{1}\right)+\beta_{*}\right) \frac{G_{m_{0}} h}{\alpha_{*}^{2}}\left(\left|\alpha^{R}\right|+\left|\gamma^{R}\right|\right)+K\left(L\left(J_{1}\right)+\beta_{*}\right) \frac{|\sigma| G_{m_{0}} h}{\alpha_{*}} .
\end{aligned}
$$
Estimates of $Q_{p}(J)$. We may assume that $m_{0}<m_{\mathcal{F}}$. It follows from (53) in Lemma
4.2 that

$$
\left|\epsilon_{p}\right| \frac{G_{m_{0}} h}{\alpha_{*}^{2}} \leq\left|\alpha_{p}^{L}\right| \frac{G_{m_{0}} h}{\alpha_{*}^{2}}+\left|\gamma_{p}^{R}\right| \frac{G_{m_{0}} h}{\alpha_{*}^{2}}+\frac{K\|G\|_{1}}{\alpha_{*}^{2}}\left\{\widehat{Q}(\Delta)+\frac{|\sigma| G_{m_{0}} h}{\alpha_{*}}\right\} .
$$

Hence, in the same fashion as Case 1 , we have

$$
Q_{p}\left(J_{2}\right) \leq Q_{p}\left(J_{1}\right)-\frac{G_{m_{0}} h}{\alpha_{*}^{2}}\left(\left|\alpha_{p}^{R}\right|+\left|\gamma_{p}^{R}\right|\right)+\frac{K\|G\|_{1}}{\alpha_{*}^{2}}\left\{\widehat{Q}(\Delta)+\frac{|\sigma| G_{m_{0}} h}{\alpha_{*}}\right\} .
$$

Estimates of $Q_{j}(J), j \neq p$. For $j>p$, we have

$$
\frac{\left|\epsilon_{j}\right|}{\alpha_{*}^{2}} \sum_{m \geq m_{0}} G_{m} h \leq \frac{\left|\alpha_{j}^{L}\right|}{\alpha_{*}^{2}} \sum_{m \geq m_{0}} G_{m} h+\frac{\left|\gamma_{j}^{R}\right|}{\alpha_{*}^{2}} \sum_{m \geq m_{0}} G_{m} h+\frac{K\|G\|_{1}}{\alpha_{*}^{2}}\left\{\widehat{Q}(\Delta)+\frac{|\sigma| G_{m_{0}} h}{\alpha_{*}}\right\} .
$$

For $j<p$, similarly,

$$
\frac{\left|\epsilon_{j}\right|}{\alpha_{*}^{2}} \sum_{m \leq m_{0}} G_{m} h \leq \frac{\left|\alpha_{j}\right|}{\alpha_{*}^{2}} \sum_{m \leq m_{0}} G_{m} h+\frac{\left|\gamma_{j}^{R}\right|}{\alpha_{*}^{2}} \sum_{m \geq m_{0}} G_{m} h+\frac{K\|G\|_{1}}{\alpha_{*}^{2}}\left\{\widehat{Q}(\Delta)+\frac{|\sigma| G_{m_{0}} h}{\alpha_{*}}\right\} .
$$

Hence, we obtain in both cases

$$
Q_{j}\left(J_{2}\right) \leq Q_{j}\left(J_{1}\right)-\frac{G_{m_{0}} h}{\alpha_{*}^{2}}\left(\left|\alpha_{j}^{R}\right|+\left|\gamma_{j}^{R}\right|\right)+\frac{K\|G\|_{1}}{\alpha_{*}^{2}}\left\{\widehat{Q}(\Delta)+\frac{|\sigma| G_{m_{0}} h}{\alpha_{*}}\right\} .
$$

Thus combining (65), (68), and (69), we obtain an analogue to (64):

$$
Q\left(J_{2}\right) \leq Q\left(J_{1}\right)-\left\{1-K L\left(J_{1}\right)-K \beta_{*}-\frac{K\|G\|_{1}}{\alpha_{*}^{2}}\right\} \widehat{Q}(\Delta)+K \frac{\|G\|_{1}|\sigma| G_{m_{0}} h}{\alpha_{*}^{3}} .
$$

Now assume that $L\left(J_{1}\right)$ and $\|G\|_{1} / \alpha_{*}^{2}$ are so small that

$$
L\left(J_{1}\right)+\beta_{*}+\frac{\|G\|_{1}}{\alpha_{*}^{2}} \leq \frac{1}{2 K}<1 .
$$


Then we have from (64) and (70)

$$
Q\left(J_{2}\right)-Q\left(J_{1}\right) \leq-\frac{1}{2} \widehat{Q}(\Delta)+\frac{|\sigma| G_{m_{0}} h}{2 \alpha_{*}}
$$

which proves the lemma in both cases.

If (71) holds for all $L\left(J_{1}\right)$ and $\beta_{*}$, by adding (59) for all interaction diamonds, it follows that

$$
\sum_{\Delta} \widehat{Q}(\Delta) \leq 2 Q(O)+\frac{2}{\alpha_{*}} \sum_{n \geq 0}\left|\sigma_{n} G_{m(n)}\right|
$$

By Lemma 4.2, the increase of $L\left(J_{1}\right)$ and $\beta_{*}$ is due to the interaction $\widehat{Q}(\Delta)$ and $|\sigma| G h / \alpha_{*}$. Hence, (71) holds for all $L\left(J_{1}\right)$ and $\beta_{*}$, provided the quantities $\alpha_{*},\|G\| / \alpha_{*}^{2}$, $L(O)$, and (55) are sufficiently small, which implies the first part of Theorem 5.1

In case we know a priori that the sign of $\sigma$ never changes, we get better estimates as follows. Assuming $\sigma \geq 0$, we define

$$
Q_{p}^{s}(J)=\frac{1}{\alpha_{*}} G_{m_{\mathcal{F}}}\left(2\left(m_{\mathcal{F}}+1\right) h-x_{\mathcal{F}}\right)+\frac{1}{\alpha_{*}} \sum_{m>m_{\mathcal{F}}} G_{m} h
$$

where the single strong shock wave crossing $J$ issues from $\left(x_{\mathcal{F}}, n k\right)$, and set

$$
\widetilde{Q}(J)=Q_{p}^{s}(J)+Q(J) .
$$

Let $J_{1}$ and $J_{2}$ be as before. If the single strong shock wave enters $\Delta$, then we find that

$$
\begin{aligned}
Q_{p}^{s}\left(J_{2}\right) & =Q_{p}^{s}\left(J_{1}\right)-G_{m_{\mathcal{F}}}\left(x_{\mathcal{F}}(n)-x_{\mathcal{F}}(n+1)\right. \\
& \leq Q_{p}^{s}\left(J_{1}\right)-\frac{\sigma_{m_{\mathcal{F}}} G_{m_{\mathcal{F}}} h}{2 \alpha_{*}}
\end{aligned}
$$

where $1 / 2$ comes from the stability condition (14). Otherwise, we have

$$
Q_{p}^{s}\left(J_{2}\right)=Q_{p}^{s}\left(J_{1}\right)
$$

Hence, we obtain, instead of (70)

$$
\widetilde{Q}\left(J_{2}\right) \leq \widetilde{Q}\left(J_{1}\right)-\left\{1-K L\left(J_{1}\right)-K \beta_{*}-\frac{K\|G\|_{1}}{\alpha_{*}^{2}}\right\}\left\{\widehat{Q}(\Delta)+K \frac{|\sigma| G_{m_{0}} h}{\alpha_{*}}\right\} .
$$

In this way, we have the following lemma which implies the second part of the Theorem.

Lemma 5.2. Let $J_{1}, J_{2}$ be two consecutive approximate space-like curves such that $J_{2}>J_{1}$ and $\Delta$ is the diamond between them. Assume that the sign of the speed of the single strong shock front never changes. If $L\left(J_{1}\right), \beta_{*}$ and $\|G\|_{1} / \alpha_{*}^{2}$ are sufficiently small, then

$$
\widehat{Q}(\Delta)+K \frac{|\sigma| G_{m_{0}} h}{\alpha_{*}} \leq 2\left(\widetilde{Q}\left(J_{1}\right)-\widetilde{Q}\left(J_{2}\right)\right)
$$




\section{Existence of global solutions}

Theorem 5.1 in the previous section implies the following uniform estimate for the approximate solutions

Theorem 6.1. Assume that $\alpha_{*}$ and $\left\|G_{1}\right\| / \alpha_{*}^{2}$ are sufficiently small and

$$
\sum_{j=1,2} \operatorname{TV}\left(\widetilde{U}_{j}(x)-\bar{U}_{j}(x)\right) \ll \alpha_{*} .
$$

1. If we assume that the quantity

$$
\sum_{n \geq 0}\left|\sigma_{n}\right| G_{m(n)} h
$$

is also small, then

$$
\operatorname{TV} U_{h, \theta}(*, t) \leq L_{0} \quad \text { for } t \geq 0 .
$$

Here the constant $L_{0}$ is independent of $h$ and $\theta$.

2. If we assume instead that the sign of the speed of the single strong shock front in the Glimm approximations never changes, then we also have the uniform estimate (79).

Once the a priori bound (79) is obtained, by repeating the argument in Glimm [3] and Liu [11], [12], we have a global solution within $L^{\infty}\left(R_{+} ; B V(R)\right) \cap L i p\left(R_{+} ; L_{\text {loc }}^{1}(R)\right)$.

In [13] and [14], Liu studies the equations of 1-D duct flow

$$
\begin{aligned}
\frac{\partial}{\partial t} \rho+\frac{\partial}{\partial x}(\rho u) & =-\frac{a^{\prime}(x)}{a(x)} \rho u, \\
\frac{\partial}{\partial t}(\rho u)+\frac{\partial}{\partial x}\left(\rho u^{2}+p\right) & =-\frac{a^{\prime}(x)}{a(x)} \rho u^{2}, \\
\frac{\partial}{\partial t}(\rho E)+\frac{\partial}{\partial x}(\rho E u+p u) & =-\frac{a^{\prime}(x)}{a(x)}(\rho E u+p u)
\end{aligned}
$$

where $\rho, u, p$, and $E$ are the density, velocity, pressure, and the total energy density of the gas, and $a(x)$ is the cross section of the duct. He shows that the transonic shock wave accelerates along a contracting duct, which corresponds to the second case of the above theorem. He also shows that the shock wave decelerates along an expanding duct, where the analysis is more delicate.

Here we can study similar phenomena in our context. Let us assume that there exists an interval $\left(x_{-}, x_{+}\right)$such that

$$
G(x, U) \equiv 0, \quad x \notin\left(x_{-}, x_{+}\right)
$$

and

$$
G(x)=o(1) \alpha_{*}^{\kappa} \quad(\kappa \geq 2) .
$$

We specify the initial data $\widetilde{U}(x)$. Let us recall the construction of standing shock waves in Section 2. We set $\widetilde{U}_{1}\left(x_{*}\right)=U_{1}\left(x_{-}<x_{*}<x_{+}\right)$and choose a constant vector $\widetilde{U}_{2}\left(x_{*}\right) \in \mathcal{S}_{p}\left(U_{1}\right)$ between $U_{1}$ and $U_{2}$ satisfying

$$
0<\sigma\left(\widetilde{U}_{1}\left(x_{*}\right), \widetilde{U}_{2}\left(x_{*}\right)\right)=O(1) \alpha_{*}^{\kappa-1-\epsilon} \quad(\epsilon>0) .
$$


Then solving the equations (7) with initial values $\widetilde{U}_{1}\left(x_{*}\right)$ and $\widetilde{U}_{2}\left(x_{*}\right)$, respectively, we define the initial data by

$$
U_{0}(x)= \begin{cases}\widetilde{U}_{1}(x), & x<x_{*}, \\ \widetilde{U}_{2}(x), & x>x_{*} .\end{cases}
$$

These initial data will satisfy the condition (13) if we choose $\widetilde{U}_{2}\left(x_{*}\right)$ arbitrarily close to $U_{2}$. Then the amount of secondary waves in $0<t<T$ is estimated as

$$
\sum_{n \geq 0} \frac{\left|\sigma_{n}\right| G_{m(n)} h}{\alpha_{*}}=o(1) \sigma \alpha_{*}^{\kappa-1} T .
$$

By choosing $T$ such that

$$
T=O(1) \alpha_{*}^{1-\kappa}
$$

the amount of secondary wave $\sin 0<t<T$ is $o(1) \sigma$ and can be made arbitrarily small compared to $\sigma$. Since the only wave crossing $O$ is the single $p$-shock wave issuing from $\left(x_{*}, 0\right)$, all weak waves are these secondary waves and their interactions. Hence we have (85) for all $0<t<T$. On the other hand, we have

$$
\sigma T=O(1) \alpha_{*}^{-\epsilon},
$$

which can be arbitrarily large. Thus we find that the single transonic shock wave goes out of the interval $\left(x_{-}, x_{+}\right)$within $0<t<T$. Hence the sign of the speed of the single strong shock front never changes and a global solution exists.

Acknowledgment. I would like to thank Professor Tai-Ping Liu for constant encouragement. I also thank the referees for helpful and encouraging comments.

\section{References}

1. I. L. Chern, Stability theorem and truncation error analysis for the Glimm scheme and for a front tracking method for flows with strong discontinuities, Comm. Pure Appl. Math. 42 (1989), 815-844.

2. R. Courant, Methods of Mathematical Physics, Wiley-Interscience, New York, 1962.

3. J. Glimm, Solutions in the large for nonlinear hyperbolic systems of equations, Comm. Pure Appl. Math. 18 (1965), 697-715.

4. J. Glimm and P. D. Lax, Decay of solutions of systems of nonlinear hyperbolic conservation laws, Amer. Math. Soc. Memoir, No.101, A.M.S., Providence, 1970.

5. J. Glimm, G. Marshall, and B. Plohr, A generalized Riemann problem for quasi-one-dimensional gas flows, Adv. Appl. Math. 5 (1984), 1-30.

6. F. John, Formation of singularities in one-dimensional nonlinear wave propagation, Comm. Pure Appl. Math. 27 (1974), 337-405.

7. P. D. Lax, Hyperbolic systems of conservation laws II, Comm. Pure Appl. Math. 10 (1957), $537-566$.

8. _ Development of singularities of solutions of nonlinear hyperbolic partial differential equations, J. Math. Phys.5 (1964), 611-613.

9. $\mathrm{Ph}$. Le Floch and P. A. Raviart, An asymptotic expansion for the solution of the generalized Riemann problem Part I: General theory, Ann. Inst. Henri Poincaré Analyse Non-linéaire 5 (1988), 179-207.

10. Li Ta-tsien and Yu Wen-ci, Boundary Value Problems for Quasilinear Hyperbolic Systems, Duke University Mathematics Series, 1985.

11. T.-P. Liu, The deterministic version of the Glimm scheme, Comm. Math. Phys. 57 (1977), 135-148.

12. — Quasilinear hyperbolic systems, Comm. Math. Phys. 68 (1979), 141-172. 
13. Nonlinear stability and instability of transonic flows through a nozzle, Comm. Math. Phys. 83 (1982), 243-260.

14. _ , Transonic gas flows along a duct of varying area, Arch. Rat. Mech. Anal. 80 (1982), $1-18$.

15. — Nonlinear resonance for quasilinear hyperbolic equation, J. Math. Phys. 28 (1987), 2593-2602.

16. J. Smoller, Shock Waves and Reaction-Diffusion Equations, Springer Verlag, New York, 1983.

Faculty of Engineering, Osaka Electro-Communication University, 18-8 Hatsucho, NeyaGAWA CITY, OSAKA 572, JAPAN

E-mail: asakura@isc.osakac.ac.jp 